UCRL-JC-125430

PREPRINT

\title{
One- and Two-Dimensional Density and Temperature Measurements of an Argon-Neon Z-Pinch Plasma at Stagnation
}

\author{
K. L. Wong, P. T. Springer, J. H. Hammer, C. A. Iglesias,
} A. L. Osterheld, M. E. Foord, H. C. Bruns, J. A. Emig, C. Deeney

This paper was prepared for submittal to the

14th International Conference on the Application of Accelerators in Research and Industry Denton, TX

November 6-9, 1996

October 24, 1996

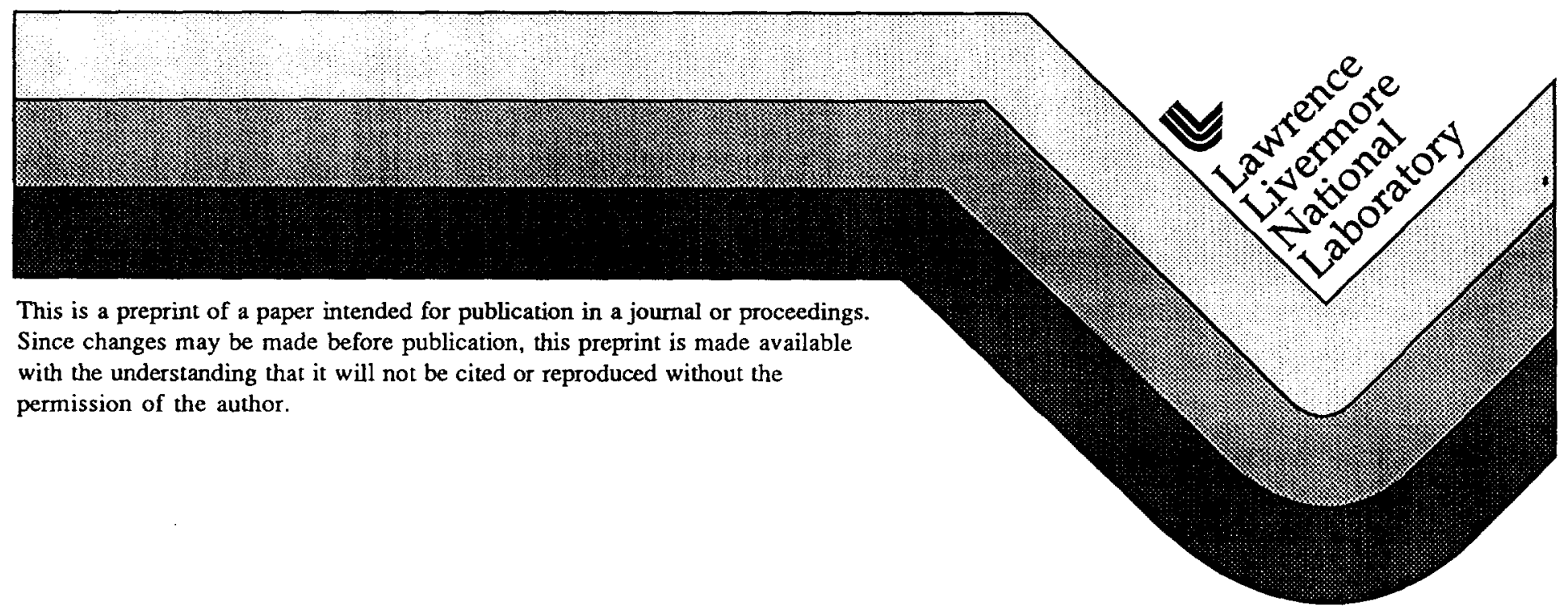




\section{DISCLAIMER}

This document was prepared as an account of work sponsored by an agency of the United States Government. Neither the United States Government nor the University of California nor any of their employees, makes any warranty, express or implied, or assumes any legal liability or responsibility for the accuracy, completeness, or usefulness of any information, apparatus, product, or process

disclosed, or represents that its use would not infringe privately owned rights. Reference herein to any specific commercial product, process, or service by trade name, trademark, manufacturer, or otherwise, does not necessarily constitute or imply its endorsement, recommendation, or favoring by the United States Government or the University of California. The views and opinions of authors expressed herein do not necessarily state or reflect those of the United States Government or the University of California, and shall not be used for advertising or product endorsement purposes. 


\title{
ONE- AND TWO-DIMENSIONAL DENSITY AND TEMPERATURE MEASUREMENTS OF AN ARGON-NEON Z-PINCH PLASMA AT STAGNATION
}

\author{
K. L. Wong, P. T. Springer, J. H. Hammer, C. A. Iglesias, A. L. Osterheld, M. E. Foord, H. C. \\ Bruns, J. A. Emig
}

Lawrence Livermore National Laboratory, Livermore, California 94551

C. Deeney

Sandia National Laboratory, Albuquerque, New Mexico 87185

\begin{abstract}
In order to benchmark and improve current 2D radiation magnetohydrodynamic (MHD) models of Z-pinch plasmas, we have performed experiments which characterize the plasma conditions at stagnation. In the experiments the SATURN pulsed power facility at Sandia National Laboratory was used to create an imploding Ar-Ne plasma. An absolutely calibrated, high resolution space- and time-resolving Johann crystal spectrometer was used to infer the electron temperature $T_{e}$ from the slope of the hydrogenlike Ne free-bound continuum, and the ion density $n_{i}$ from the Stark broadening of the Ar heliumlike Rydberg series. 2D electron temperature profiles of the plasma are obtained from a set of imaging crystals also focussed on the Ne free-bound continuum. We shot two types of gas nozzles in the experiment, annular and uniform fill, which varies the amount of mass in the plasma. 2D local thermodynamic equilibrium (LTE) and non-LTE MHD models predict a radiating region denser and cooler than measured.
\end{abstract}

\section{INTRODUCTION}

$\mathrm{Z}$-pinch accelerators have been used for many years as $\mathrm{x}$ ray sources ( $P>1 \mathrm{TW}, \mathrm{E}<3 \mathrm{keV}, \mathrm{t} \sim 40 \mathrm{~ns}$ ) for applications such as materials testing and $\mathrm{x}$-ray lithography and microscopy(1). However, due to the complexity of the pinch dynamics these sources have not been modeled from first principles. Furthermore, direct comparison with experiment has been difficult because of a lack of high precision data (poor resolution, time integrating spectrometers). Therefore, there is clearly a need for high resolution, time-gated diagnostics which can be used to test current 2D radiation MHD models(2).

In this paper we present the first high-resolution, timeresolved measurements of a $Z$-pinch plasma. The purpose of this experiment was to provide high quality data on the plasma conditions to compare with models. The goal would be to provide a predictive capability in order to optimize x-ray output and to scale the output to larger future sources.

\section{EXPERIMENT}

The SATURN accelerator located at Sandia National Laboratory in Albuquerque, $N M(3)$ was the $x$-ray source used in the experiment. The peak power of the generator was $15 \mathrm{TW}$. Hot $\mathrm{Ne}$-Ar plasmas are formed by injecting $600 \mu \mathrm{g}$ annular or $200 \mu \mathrm{g}$ uniform $90 \% \mathrm{Ne}-10 \%$ Ar neutral gas columns into the $5 \times 10^{-5}$ torr vacuum between the anode and cathode of the device. A pair of UV flashboards preionize the gas, and SATURN is fired $1 \mu$ s later which delivers $\sim 8 \mathrm{MA}$ at $1.9 \mathrm{MV}$ to the load. The $\mathrm{J} X \mathrm{~B}$ force causes the plasma to implode at $\mathrm{v}=10^{8} \mathrm{~cm} / \mathrm{s}$. The plasma stagnates or reaches the cylindrical axis in $60-90 \mathrm{~ns}$, and a burst of $x$-rays is released as the plasma kinetic energy is transferred to $x$-rays.

A high resolution space- and time-resolving Johann crystal specrtometer was used to monitor the $x$-rays in the experiment. The spectrometer is equipped with three 100 $\mu \mathrm{m}$ vertical inaging slits which radially image the source with a magnification of 1.7. The data were taken using the spectrometer with four separate crystals. We used $10 \mathrm{~cm}$ long $A D P(101)$ and quartz(1120) crystals with lattice spacings of $2 d=10.64 \AA$ and $4.912 \AA$, respectively. The crystals were bent to radii of curvature of $50 \mathrm{~cm}$ and the $\mathrm{x}$ rays were spectrally dispersed and focussed at the $25 \mathrm{~cm}$ Rowland circle radius. The resolving power of the setup is $\mathrm{E} / \Delta \mathrm{E}=3000$. The spectrometer was set to a nominal Bragg angle of $40^{\circ}$. The total $x$-ray energy range covered was 1.6-2.0 keV for the ADP crystal and 3.4-4.4 keV for the quartz crystal. In order to obtain $2 \mathrm{D}$ images of the plasma we used $1 \mathrm{~cm}$ long $\mathrm{ADP}(101)$ and quartz(1120) crystals bent to radii of curvatute of $88 \mathrm{~cm}$. Crystals bent to this radius produce monochromatic images of the plasma at the $25 \mathrm{~cm}$ Rowland circle in the spectral direction. The Bragg angles for the ADP and quartz crystals were set independently to $35^{\circ}$ and $42.5^{\circ}$, respectively. The ADP and quartz imaging crystals had a demagnification of 7.7 and 4.6, repectively in the axial direction. A schematic of the experimental setup is shown in Fig. 1 . We record the $x$ rays on a two-dimensional gated microchannel plate (MCP) detector with three active striplines which can be biased to different voltages coupled to a phosphor coated fiber optic plate. The MCP, coated with a Au photocathode, is active for $1 \mathrm{~ns}$ of the typical $40 \mathrm{~ns}$-ray pulse of SATURN. The 


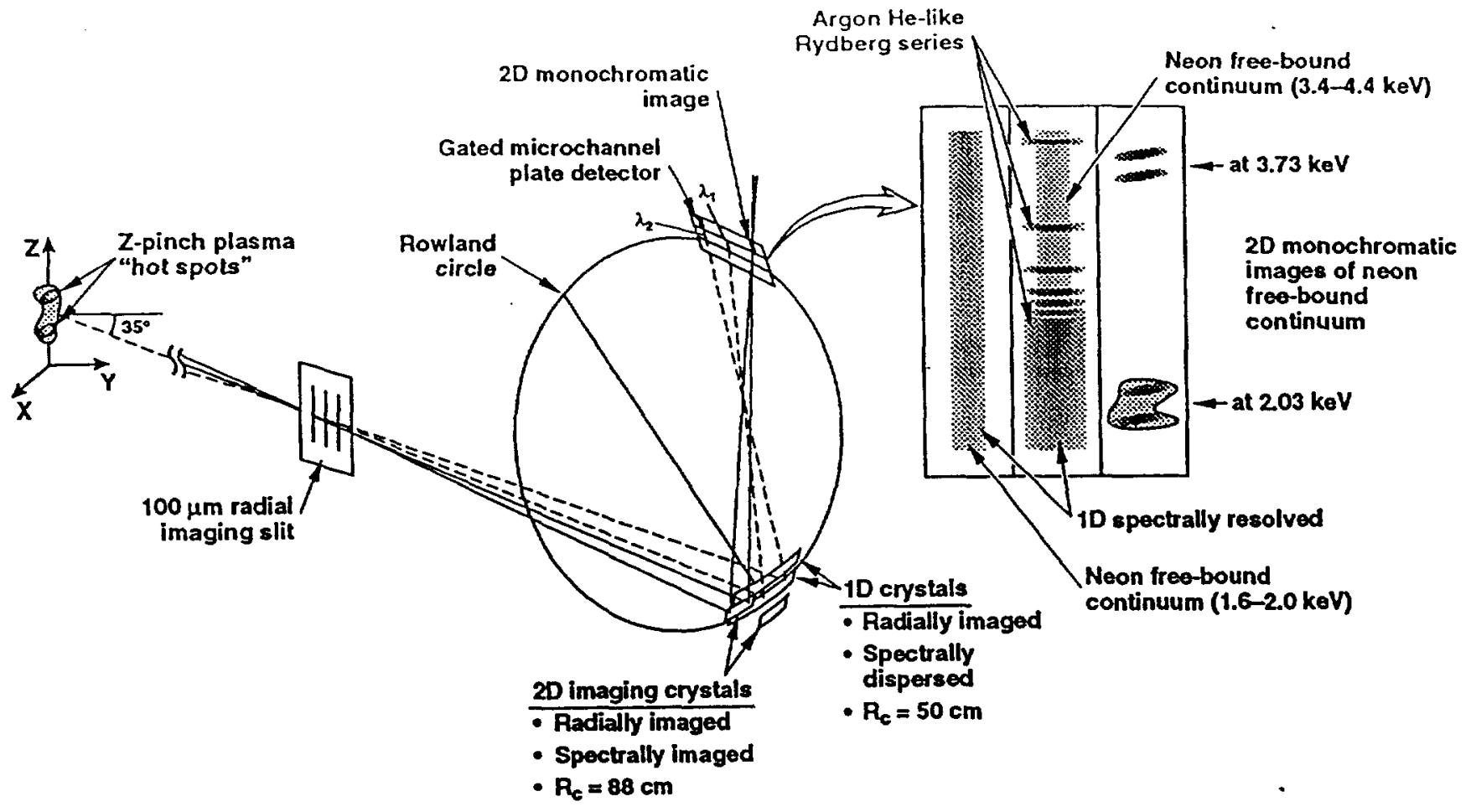

FIGURE 1. Schematic drawing of the experimental setup.

data were recorded on Kodak optical TMAX film. A microdensitometer was used to measure the film optical density versus position. Each piece of film was developed with a preexposed calibrated step wedge to convert optical density to x-ray exposure.

Fig. 2 shows a typical piece of film data from an Ar-Ne shot. The broad band exposure running across the top strip from the ADP crystal is radiation due to recombination onto bare neon ions resulting in the hydrogenlike free-bound continuum from 1.6-2.0 keV. The bright lines running vertically on the strip are 2 nd order Ar Rydberg series lines
He-like(1-3), He-like(1-4), and H-like(1-3). The lines on the middle strip illuminated by the quartz crystal are the Heand H-like Ar Rydberg series. We mesure form the Helike(1-3) to the He-like(1-9) and from the H-like(1-3) to the H-like(1-9). Superimposed on the Rydberg series is the hydrogenlike neon free-bound continuum radiation from 3.4-4.4 $\mathrm{keV}$. The bottom strip shows the monochromatic $2 \mathrm{D}$ images of the plasma from the ADP (left) and the quartz (right) crystals of the hydrogenlike neon-free bound continuum at $2.03 \mathrm{keV}$ and $3.73 \mathrm{keV}$, respectively.

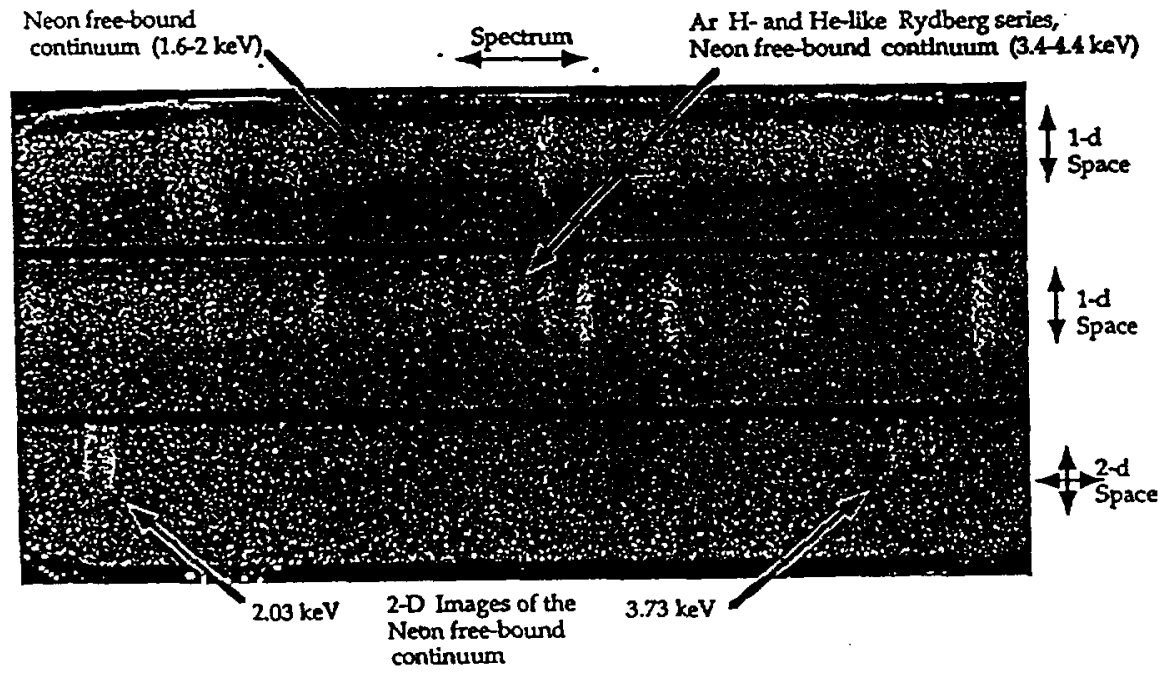

FIGURE 2. A typical piece of film data showing radially resolved spectra of an $10 \%$ Ar- $90 \%$ Ne z-pinch plasma in the top two strips. The bottom strip shows two-dimensional monochromatic images of the plasma. 


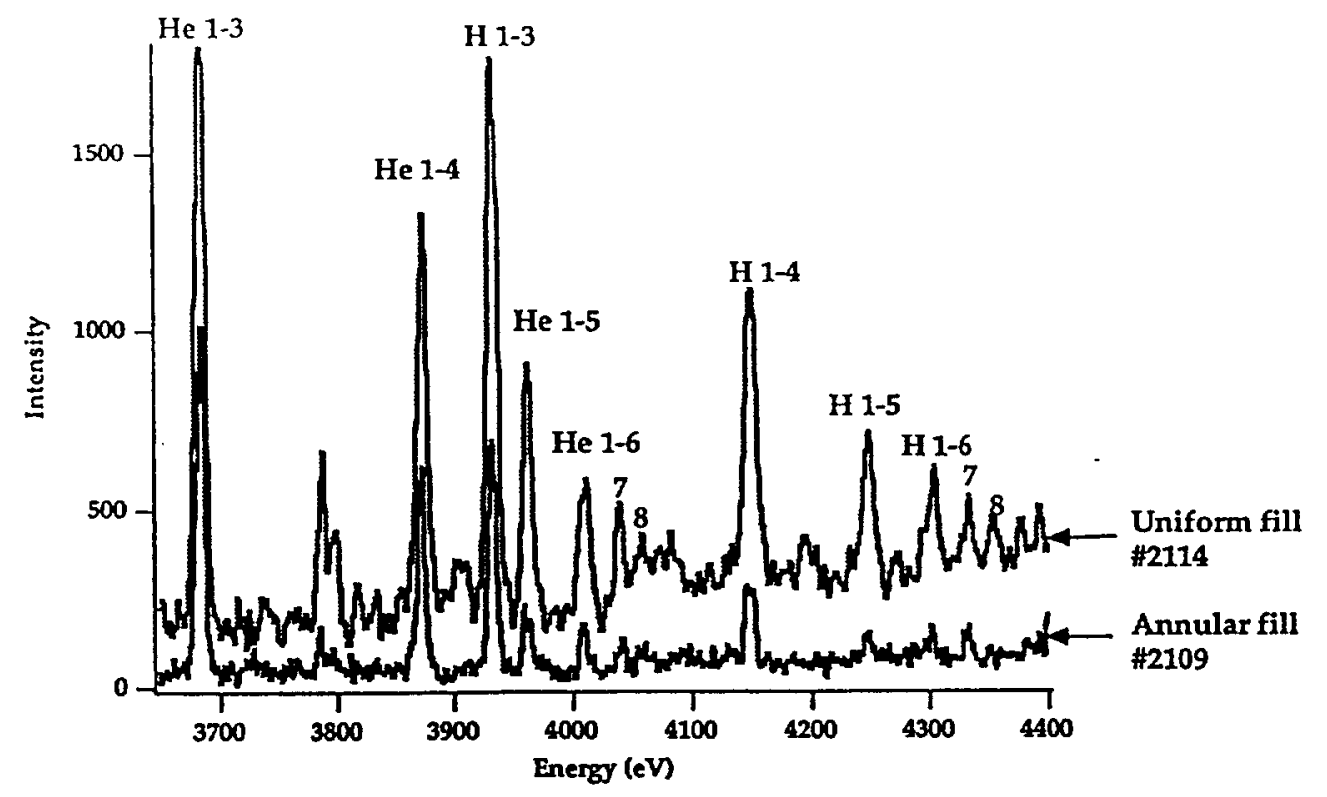

FIGURE 3. Heliumlike and hydrogenlike Ar Rydberg series for uniform and annular fill z-pinch plasmas.

\section{RESULTS}

A plot of the heliumlike and hydrogenlike Rydberg series is shown for both a uniform and annular gas puff in Fig. 3. The spectra is obtained by taking a horizontal projection of the data on the center strip as in Fig. 2. The uniform fill plasmas which had less mass and were less dense implode at a faster speed, stagnate on axis with a higher electron temperature, and reach a higher ionization balance. This was evident from the data in Fig. 3 showing that the uniform fill gas puffs had a higher ratio of the $\mathrm{H}$-like to $\mathrm{He}$ like Ar Rydberg series.

The electron density $n_{e}$, ion density $n_{j}$, and ion temperature $T_{i}$ are inferred from a fit to the He-like Ar Rydberg series. We performed a two component Gaussian least-squared fit to the data. One component was assumed to be due to a constant Doppler line broadening and the other due to Stark broadening in which the width of the lines was assumed to vary as $N^{2} n_{e}{ }^{2 / 3}$ where $N$ is the principle quantum number of the upper level in the Rydberg transition. From the Doppler width we interpreted the ion temperature to be $36 \mathrm{keV}$, but this may also be evidence of turbulence or mass motion. To infer the electron density we compared the experimental Stark width from the fit to that computed by the TOTAL calculation(4). TOTAL is a code in which the Stark broadening of line radiation in a plasma can be determined from the electric microfields produced at the radiator by ion and electron perturbers. We determined an electron density of $0.8 \times 10^{21} \mathrm{~cm}^{-3}$ for the annular gas puff. Assuming this electron density and a plasma consisting of $90 \% \mathrm{Ne}^{10+}$ and $10 \% \mathrm{Ar}^{16+}$, the density of the plasma is $0.0028 \mathrm{~g} / \mathrm{cm}^{3}$.
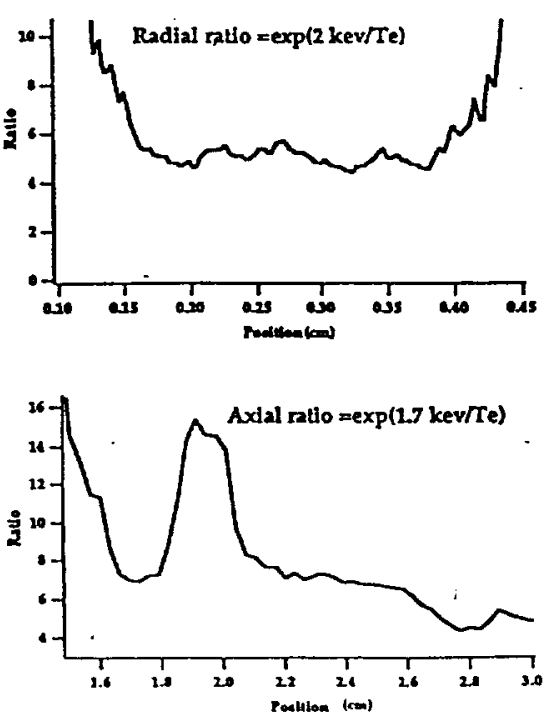

FIGURE 4. One- and two-dimensional electron temperature maps can be determined from spectra and monochromatic images of the $\mathrm{Ne}$ free-bound continuum near 2 and $4 \mathrm{keV}$. 
The $2 \mathrm{D}$ electron temperatures $\mathrm{T}_{\mathrm{e}}$ are determined from the slope of the hydrogenlike $\mathrm{Ne}$ free-bound continuum radiation for these optically thin plasmas. The slope is computed by taking the ratio of the ADP and quartz $x$-ray intensities $I(E)$ and $I(E+\Delta E)$, respectively, where $E$ is the photon energy and using the $\mathrm{I}(\mathrm{E}) /(\mathrm{E}+\Delta \mathrm{E})=\exp (\Delta \mathrm{E} / \mathrm{kT}$ e $)$ intensity fall off of a Maxwellian electron velocity distribution. The intensity ratios are corrected for absolute spectrometer efficiency of each crystal, Be-filter transmission, MCP detector gating response, film sensitivity, $x$-ray transmission through the gas column, and the magnification difference for the ADP and quartz imaging crystals. The spectrometer was absolutely calibrated using $\mathrm{Au}$ bremsstrahlung $\mathrm{x}$-rays produced by a Manson source. We measured the diffracted $x$-rays on the detector using a single photon counting photomultiplier tube (PMT) system relative to the direct $\mathrm{x}$-ray flux monitored by a windowless lithiun-drifted silicon $\mathrm{Si}(\mathrm{Li})$ detector. The radial temperature profiles were determined by taking radial projections of the Ne free-bound radiation from the ADP crystal data (top strip in Fig. 2) at an x-ray energy of $1.7 \mathrm{keV}$ and from the quartz crystal at $3.7 \mathrm{keV}$ (middle strip in Fig. 2). Similarly, the axial temperature profiles were inferred from axial projections of the 2D monochromatic Ne free-bound images from the ADP and quartz imaging crystals at $x$-ray energies of $2.03 \mathrm{keV}$ and $3.73 \mathrm{keV}$, respectively, as seen on the bottom strip in Fig. 2. The results are shown on Fig. 4. We measured peak radial and axial electron temperatures of $1.2 \mathrm{keV}$ for the annular fill plasma and $1.6 \mathrm{keV}$ for the uniform fill plasma.

We have compared our results to both 2D local thermodynamic equilibrium (LTE) simulations(2) where the level populations are statistically populated and 2D nonLTE calculations where the populations are determined by rate equations. The predicted $x$-ray pulse widths and total radiation yields are in reasonable agreement with the measurements. However, the models also give higher densities, smaller radii, and lower temperatures at stagnation when compared with the experiment. The 2D LTE calculation predicts a stagnation density of $\sim 1 \mathrm{~g} / \mathrm{cm}^{3}$ and the full width at half maximum (FWHM) of the pinch to be $\sim$ microns vs. $2 \mathrm{~mm}$ for the experiment with an electron temperature of $300 \mathrm{eV}$. The $2 \mathrm{D}$ non-LTE model gives an improved stagnation density of $\sim 10^{-2} \mathrm{~g} / \mathrm{cm}^{3}$ with a FWMH pinch of $0.5 \mathrm{~mm}$ and an electron tempetature of $700 \mathrm{eV}$. In these models in which the plasma cools by radiating during the implosion the final predicted temperatures are too low to produce the H-like Ar ionization state seen in the measurement. The discrepancies could be due to $3 \mathrm{D}$ effects such as additional angular momentum breaking the azimuthal symmetry and turbulence in the equation of state which both would prevent the plasma from reaching the high densities at stagnation.

In conclusion, we have performed the first high resolution time-resolved measurements of the plasma conditions of a Z-pinch accelerator at stagnation. 2D electron temperature profiles, electron densities, ion temperatures, and ion densities were inferied to compare with 2D radiation MHD models. We find the simulations predict plasmas that are too dense, tightly pinched, and cool at stagnation. Possible
3D effects and adding turbulence may explain these discrepancies.

\section{ACKNOWLEDGEMENTS}

We wish to thank A. Toor and M. Eckart for their support of the experiment, and the SATURN PRS crew for their technical assistance. This work was performed under the auspices of the U. S. Department of Energy by Lawrence Livermore National Laboratory and Sandial National Laboratories under contracts W-7405-ENG-48 and DEAC04-94AL85000.

\section{REFERENCES}

1. Pereira, N. R. and Davis, J., J. Appl. Phys. 64, p. R1 (1988).

2. Hammer, J. H. et al, Phys. Plasmas 3, p. 2063 (1996).

3. Bloomquist, D. D. et al., Proceedings of the 6th Institute of Electrical and Electronics Engineers Pulsed Power Conference, Arlington, VA, 1987, p. 310.

4. Calisti, A. et al., Phys. Rev. A 42, p. 5433 (1990). 


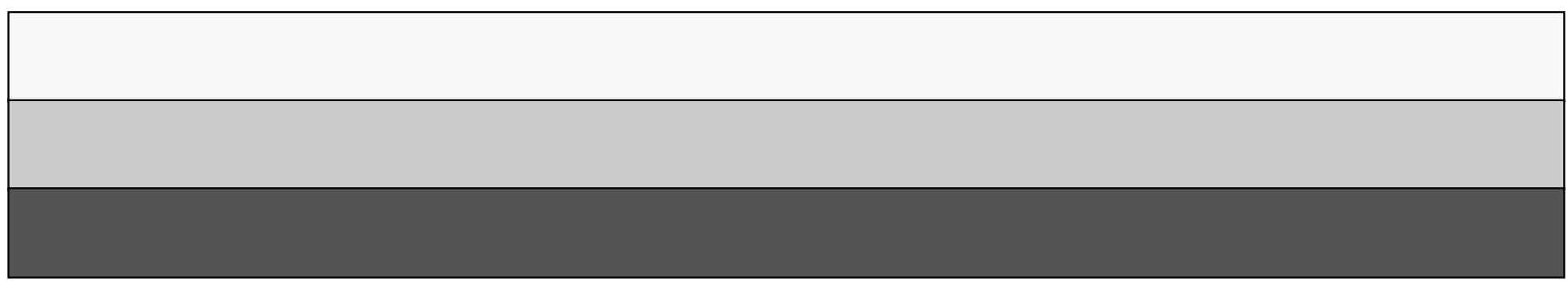

